\title{
Genome-Wide Identification and Functional Analysis of U-Box E3 Ubiquitin Ligases Gene Family Related to Drought Stress Response in Chinese white pear (Pyrus bretschneideri)
}

Chunmeng Wang

Nanjing Agricultural University - Weigang Campus: Nanjing Agricultural University

Bobo Song

Nanjing Agricultural University - Weigang Campus: Nanjing Agricultural University

Yuqin Dai

Nanjing Agricultural University - Weigang Campus: Nanjing Agricultural University

Shaoling Zhang

Nanjing Agricultural University - Weigang Campus: Nanjing Agricultural University

Xiaosan Huang ( $\nabla$ huangxs@njau.edu.cn )

Nanjing Agicultural University

\section{Research article}

Keywords: ubiquitin ligases, PUB gene family, Pyrus betulaefolia, PbrPUB18, abiotic stresses

Posted Date: February 10th, 2021

DOI: https://doi.org/10.21203/rs.3.rs-201827/v1

License: (c) (1) This work is licensed under a Creative Commons Attribution 4.0 International License.

Read Full License 


\section{Abstract}

\section{Background}

The plant U-box(PUB) proteins are a family of ubiquitin ligases (E3) enzymes that involved in diverse biological processes, as well as in responses to plant stress response. However, the characteristics and functional divergence of the $P U B$ gene family has not yet been previously studied in the Chinese White Pear (Pyrus bretschneideri).

\section{Results}

In the present study, we identified 62 PbrPUBsin Chinese white pear genome. Based on the phylogenetic relationship, 62 PUB genes were clustered into five groups. The conserved motif and gene structure analysis provided further evidence to support the classification phylogenetic tree. The PbrPUB genes were unevenly distribution on 17 pear chromosomes, chromosome 15 housed most member of $P U B$ family, with eight $P U B$ genes. Cis-acting element analysis indicted $P U B$ genes might participate in diverse biological processes, especially in the response to abiotic stresses. Based on RNA-data from "Dangshansuli" at seven tissues, we found that PUB genes exhibited diverse of expression level in seven tissues, and qRT-PCR experiment further support the reliable of RNA-Seq data. To identify candidate genes associated with resistance, we conducted qRT-PCR experiment the expression level of pear seed plant under four abiotic stresses, including: $A B A$, dehydration, salt and cold treatment. One candidate PUB gene associated with dehydration stress was selected to conduct further functional experiment. Subcellular localization revealed $P b r P U B 18$ gene was located on cell nucleus. Furthermore, heterologous over-expression of PbrPUB18 in Arabidopsis indicated that the over-expression of PbrPUB18 could enhance resistance in drought treatment. In conclusions, we systematically identified the $P U B$ genes in pear, and provided valuable information for the molecular mechanism of $P U B$ genes in pear.

\section{Background}

Plants are frequently exposed to various abiotic stresses such as drought, salt and low temperature during their life cycles. Several stresses often lead to oxidative damage and have adverse impacts on plant growth and development. To adapt to unfavorable environmental conditions, plants have evolved complex and efficient mechanisms [1]. Previous studies have identified four signal transduction pathways in response to abiotic stress, including transcriptional regulation, post-transcriptional modifications, epigenetic regulation, and post-translational modifications [2]. And ubiquitination is one of the most significant post-translational modifications. The ubiquitin/26S proteasome system (UPS) pathway is a pervasive and effective route for protein removal in eukaryotes [3, 4]. UPS include ubiquitin $(\mathrm{Ub})$, ubiquitin-activating enzyme (E1), ubiquitin- conjugating enzyme (E2), ubiquitin ligase (E3), and the $26 \mathrm{~S}$ proteasome. The central component of UPS is the highly conserved, 76 amino acid protein ubiquitin. Ubiquitin is bound to specific proteins and functions in the degradation of target proteins in an E1-E2- 
E3 multienzyme cascade manner [5-8]. In the pathway, E3 enzymes are clearly the key factors that define substrate specificity. According to their reaction mechanism and subunit compositions, four main types were classified: Ubox, HECT (Homology to E6-Associated Carboxy-Terminus), RING (Really Interesting New Gene) and Cullin-RING ligases (CRLs)[4]. U-box ubiquitin ligases are characterized by a conserved U-box motif of about 70 amino acids. And U-box ubiquitin ligases were firstly discovered among E3 ubiquitin ligases, and was first clarified from ubiquitin fusion degradation protein-2 (UFD2) in yeast [9].

In comparison with the 2 and 21U-box (PUB) genes identified in Saccharomyces cerevisiae and Homo sapiens genomes, respectively, more U-box genes were widely distributed in plants. In Arabidopsis thaliana, about 61 plant U-box genes were predicted [9, 10], while 77 were found in Oryza sativa [11], 62 in Solanum lycopersicum [12], 93 in Gossypium raimondii [13], 91 in Musa acuminate [14], 61 in Medicago truncatula [15] 101 in Brassica rapa [16] and 125 in soybean [17]. Many previous studies have shown that PUB proteins are involved in biological processes such as plant hormone signaling regulations [6], selfincompatible or pseudo-self-compatibility regulations [18] a as well as in biotic stress [19-21] and abiotic stress $[5,22,23]$.

In a number of previous studies, U-box genes acted as regulators in diverse abiotic stress responses including drought, low temperature and salinity conditions. In Arabidopsis thaliana, AtPUB18/AtPUB19 are negative regulators of $A B A$ signaling by inducing $A B A$ hypersensitivity, and AtPUB22/AtPUB23 are negative regulators in drought stress responses in an ABA-independent pathway [24, 25]. PUB25 and $P U B 26$, two U-box typeE3 ubiquitin ligases, trigger cold signaling negative regulator MYB15 to promote plant freezing tolerance[26]. AtPUB44 ubiquitinates the AAO3 (abscisic aldehyde oxidase 3 ) via 26 proteasome and affects the ABA biosynthesis [27]. Furthermore, AtPUB46 and AtPUB48 were found to be more sensitive to drought [28]. In rice, OSPUB15 has been implicated in positive regulating plant tolerance to salinity and drought stress [29]. In apple, MdPUB29 may positively regulate salt tolerance [30].

The plant PUB family has been widely studied for abiotic stresses, mainly in model plants such as Arabidopsis, rice and tomato, and less on woody plants such as pear. Pear belongs to the Pyrus genus in the Rosaceae family, and is one of the most important fruit crops and widely distributed fruits in the world. However, the yield of pear frequently fell off on account of abiotic stress such as drought, cold and salinity. And these affect pear growth and development, furthermore limit pear crop productivity [31]. Therefore, it is significant to identify genetic determinants associated with drought, cold and salinity stresses tolerance in pear for agricultural development. The completion of Chinese White Pear (Pyrus bretschneideri) genome sequencing has provided a golden opportunity to study the function of the pear U-box proteins at the whole genome scale [32]. In this study, we conducted systematic identification of PUB genes in pear on whole-genome level, and further chromosome location, genomic structure, evolutionary analysis and experiment verification will establish a solid foundation for functional characterization of $P b r P U B$ genes in the future.

\section{Results}




\section{Identification of PbrPUB gene family members}

In our study, we used a strictly pipeline to identify PUB genes in pear genome. First, the hidden Markov model (HMM) of the U-box domain (PF04564) was downloaded from the Pfam30.0 database, and used as a query to identify the candidate PUB members in Chinese white pear genomic database (http://peargenome.njau.edu.cn/) usingHMMER3.0. As a result, a total of 91 candidate PUB genes were identified in pear genome. Second, SMART tools were performed to verify the accuracy of 91 candidate PUB gens, and 29 PUB genes were removed because of the incomplete of PUB domain. At last, 62 PUB genes with complete U-box domain were obtained for further analysis (Table 1). We named these PbrPUB genes from PbrPUB1 to PbrPUB62 according to their location information on the chromosome. The ExPASy ProtParam (https://www.expasy.org/), an online proteomics and sequence analysis tool, was used to estimate molecular weight and isoelectric point (pl). The molecular weight for the PbrPUB gene family range from $39.33 \mathrm{kDa}$ to $151.30 \mathrm{kDa}$ (Kilodalton) and the pl range from 4.99 to 8.83 , with an average of 6.78. Subcellular localization were also predicted by Cell-PLoc 2.0 (http://www.csbio.sjtu.edu.cn/bioinf/Cell-PLoc-2/), and we found that most PUB gene were located in nucleus, except six pub located in cytoplasm and three located in cell membrane.

\section{Phylogenetic analysis of PbrPUB gene family members}

To investigate the evolutionary relationships of $P b r P U B$ genes family, we constructed a neighbor joining $(\mathrm{NJ})$ phylogenetic tree using the Mega-X program based on the full-length PUB proteins of pear (62 members), tomato (62 members) and Arabidopsis (61 members) (Fig. 1a). The protein sequences of PUB genes of tomato and Arabidopsis were obtained from previous study [10,12]. Based the result of phylogenetic tree, 185 members of PUB genes from these three species were clustered into five groups, including Group I, Group II, Group III, Group IV and Group V. The member number of Group III was biggest in five subgroups, and it harboured 64 PUB genes. However, Group IV harboured least PUB genes, with 10 PUB genes. In generally, the PUB genes of pear and tomato were clustered into one subclade, suggesting that pear and tomato exhibited relatively closer relationship compared to Arabidopsis.

It is interesting to note that the member number of PUB gene family in these three species is similar. This result indicated that the number of PUB genes in these three species is conserved. To explore which group of pears had occurred expansion or lost during evolution process, we measure the number of PUB genes of each species in each group. In pear, group I, II, III, IV and V contain 11, 21, 21, 3 and 6 PbrPUB gene family members, respectively. In tomato, groups II II, III, IV and V contain 12, 21, 21, 3, and 5 SIU-box genes, respectively. In Arabidopsis, groups I, II, III, IV and V contain 20, 12, 22, 4 and 3 AtPUB genes, respectively (Fig. 1b). The member number of each group in pear and tomato is almost equal, suggesting that pear had not undergone expansion or lost compared to tomato. However, compared to pear and tomato, the group I of Arabidopsis had undergone rapid expansion, while the group II of Arabidopsis had undergone rapid lost. 
To further verify the classification results of phylogenetic tree, we investigated the conserved motif and gene structure of PbrPUB genes in pear. The MultipleEm for Motif Elicitation (MEME) motif search tool were used to predict the conserved motif of $P b r P U B$ genes. A total of 20 motifs were estimated in our study, and we named as motif 1-20 (Fig. 2a, 2b, Additional file 1: Fig. S1). Among them, motif 1, 3 and 5 were found in all groups, indicating that were highly conserved in all PbrPUB proteins. Based on the SMATR website, we determined that the U-box was comprised of Motif1, Motif3 and Motif5 (Additional file 2: Fig.S2). This result provided evidence to support the accuracy of PUB gene set identified in our study. Based on the SMATR website, we also found the other conserved domain: ARM and Pkinase domin. The ARM is comprised of motif 2, 4 and 7; the Pkinase is comprised of motif 11, 13 and 20.Generally, most PbrPUB members in the same groups had similar motifs. For example, most of the members of group II contained motif 6,10 and 8 . This result indicated that these three motifs might be key functional domain of group II PUB genes, suggesting that these proteins might have conservative functions.

To explore the gene structure of $P b r P U B$ genes in pear, we extracted the exon-intron information of 62 $P b r P U B$ genes from pear database using in-house scripts. Based on the information, TBtools software were preformed to show the gene structure of $P b r P U B$ (Fig. 2C). The number of exon in PUB genes was greatly divergent, ranging from 1 to 20 . Among 62 PUB genes in pear, PbrPUB24 contained the greatest number of exon (20), while 16 PUB genes only contained one exon. Furthermore, the lengths of the exon and intron were differential. There are 30 PUB genes have been found contain UTR structure. Similarly, to the result of motif analysis, the PUB genes with similar gene structure were cluster into same subclade. For example, most members of class II only housed one exon. This result indicated that the members of same groups exhibited similar gene structure and conserved motifs. These results from conserved motifs and gene structure analysis provided strong evidence to support the accuracy of the classification result of phylogenetic tree.

\section{Chromosomal localization and homologous gene analysis of PbrPUB genes}

To further explore the distribution pattern of PUB gene in pear genome, the PbrPUB chromosomal distribution map was plotted using TBtools (Fig. 3a). The location information of PUB genes in pear were extracted by our in-house scripts. As a result, a total of 50 PbrPUB genes (82.26\%) were unevenly mapped on the 17 pear chromosomes, and no member of $P b r P U B$ gene family was mapped on chromosome 8. Therefore, we didn't shown chromosome 8 in our Fig. 3. In addition, 12 genes were located on scaffold contigs, and we also didn't show them in our Fig. 3. Chromosome 15 had the largest number of PbrPUB genes, with eight $P b r P U B$ genes, followed by chromosome 5 with 6 genes. Chromosome 1, 2 and 12 each contained 4 PbrPUB genes. Two or three PbrPUB genes were mapped on chromosomes 3, 6, 7, 9, 10, 11, 13,14 , and 16 . Chromosome 4 and chromosome 17 contained only one gene. We also identified the homologous genes of PUB gene family using MCscanX software. As result, 16 homologous gene pairs were identified in pear PUB gene family, which contained 26 homologous genes. Three homologous gene pairs were detected between chromosomes 5 and chromosomes 10 (Fig. 3b). 


\section{Cis-acting elements analysis in the putative promoter of PbrPUB genes}

Cis-acting elements were important clues for the prediction of gene functions. To further investigate the function of $P b r P U B$ genes, we predicted the cis-acting element of the putative promoter region of $P b r P U B$ genes using PlantCARE databse. In this study, the 2000 bp upstream region of transcription initiation site of $P b r P U B$ genes was defined as the putative promoter region. As a result, a tot al of 41 cis-acting elements were identified (Fig. 4), and we selected 15 interesting cis-acting elements for further analysis. These 15 cis-acting were associated with stress, hormone, plant growth and development. As shown in Fig. 4a, some diverse distribution patterns of cis-acting elements were observed in the promoter region of PbrPUB genes, indicating that the PUB gene family of pear particular in various different biology process. Meanwhile, we found that all PbrPUB genes contained the cis-acting related to hormone regulation, such as, abscisic acid (ABA), methyl jasmonate (MeJA), gibberellin (GA), salicylic acid, and auxin responsiveness elements. Previous study had reported that $D S G 1$, which encodes a U-box domain, could regulate cell division and elongation by responding to multiple hormones, such as auxin, salicylic acid and ethylene [33]. In our study, 55 genes were identified as the responsiveness elements of ABA, suggesting that PUB gene family might particular in resistance under ABA treatment (Fig. 4b). In Arabidopsis, AtPUB9, AtPUB18, AtPUB19, and AtPUB44 were identified to involve in ABA response [34]. It is notable that the element related to MYB binding site involved in drought was predicted in $44 \mathrm{PbrPUB}$ genes, suggesting that these 44 PbrPUB genes might mediated by MYB genes response to drought stress. Moreover, there were 30 PbrPUB genes have cis-acting elements related to cold, suggesting that these 30 PbrPUB genes might particular in resistance under low temp treatment. As we all know, flavonoid biosynthesis is one of important phenomenon during the process of response to stress in plant. In the study, we found PbrPUB10, PbrPUB24 and PbrPUB5 contained MYB binding site involved in flavonoid biosynthetic.

\section{Expression profile of PbrPUB genes in different tissues of pear}

To further explore the tissues-specific expression of PbrPUB genes, we conducted RNA-seq analysis using transcriptomic data from seven different tissues of 'Dangshansuli' cultivar pear, including stem, ovary, petal, sepal, bud, fruit and leaf [35]. We used RPKM (reads per kilobase per million) values to estimate the expression level of PbrPUB genes. The RPKM value equal to 0 means no expression in one library, and the RPKM value greater than 10 means highly expression in one library. Then, we investigated the expression level of 62 PUB genes. Pheatmap, an R package, was used to show the expression patterns of 62 PbrPUB genes (Fig. 5a). Based on the expression pattern of 62 PUB genes, they were clustered into four main classes. Gene in Class IV exhibited highly expression level in all of seven examined tissues, while gene in Class II exhibited almost no expression in all of seven tissues. Class I was specifically expressed in pear leaf, and a diversity of expression pattern were detected in Class III (Fig. 5a). Among the 62 PbrPUB genes, 52 genes (83.87\%) were at least expressed in one tissue, even though the transcript abundance of several genes was relatively lower for certain tissues. Approximately 10 non-expressed PUB genes were identified in all of seven tissues, and they may be lost the function during the evolution process of PUB genes family in pear. 29 PbrPUB genes were expressed in all seven different tissues, indicating that they 
have various roles in the development of different tissues. Interesting, we found 28 PUB genes exhibited highest expression in leaf, suggesting that these 28 genes might involve the development of leaf. Due to leaf is an important plant organ involved resistance, we referred that these 28 PUB genes might particular in resistance in the process of pear growth and development.

To verify the transcriptome sequences analysis was reliable, $15 \mathrm{PbrPUB}$ genes were randomly selected to conduct a quantitative real-time PCR (qRT-PCR) experiment to investigate the expression levels in seven different tissues of the 'Dangshansuli' pear, including stem, leaf, petal, fruit, sepal, bud, and ovary (Fig. 5b). We found that all of 15 PbrPUB genes exhibited a diversity of expression patterns in the seven different tissues, suggesting that $P b r P \cup B$ genes family may function in different tissues and participate in diverse metabolic processes. Seven genes ( $P b r P U B 1, P b r P U B 3$, PbrPUB7, PbrPUB9, PbrPUB18, PbrPUB36 and PbrPUB38) exhibited a similar expression pattern with a high expression level in leaf tissues, suggesting that $P b r P U B$ genes play critical functions during leaf development. All of these seven genes exhibited highly expression level in leaf in transcriptome data. These result provided further evidence to support our transcriptome sequences analysis was reliable. Interestingly, most of 15 PUB genes were highly expressed in reproductive organ, suggesting that $P b r P \cup B$ genes might associate with the development of reproductive organ.

\section{The expression pattern of PbrPUB genes under abiotic stresses}

Previous study had extensively reported $P b r P U B$ gene family involved in various abiotic stresses [36]. To explore the function of PUB gene family in pear, Pyrus betulaefolia from grown 45-day-old seedlings were subjected to four different stress treatments including dehydration, low temperature, ABA and salt. To identify whether PUB genes in pear could respond to abiotic stresses, 11 PbrPUB genes were randomly selected from each group to conduct a qRT-PCR experiment to detect their expression level. 11 genes are comprise of 2 from group I (PbrPUB1 and PbrPUB14), 4 from group II (PbrPUB12, PbrPUB18, PbrPUB36 and PbrPUB38), 2 from group III (PbrPUB3 and PbrPUB25), 2 from group IV (PbrPUB7 and PbrPUB48) and 1 from group $\mathrm{V}(\mathrm{PbrPUB34})$.

To identify candidate $P b r P U B$ genes related to dehydration treatment, the shoots of seedlings of pear were placed on dry filter papers for 0, 1, 6, 9, 12 and $24 \mathrm{~h}$ at ambient environment (Fig. 6a). Among the eleven PUB genes, 9 PUB genes were up-regulated expressed and one PUB gene (PbrPUB 7 ) was downregulated expressed under dehydration stress. However, PbrPUB14 was not significantly differential expressed under dehydration stress. Among the 9 up-regulated genes, PbrPUB18 exhibited highly increased expression level during the process of dehydration treatment, while PbrPUB12, PbrPUB3 and $P b r P U B 36$ were up-regulated expressed during $12 \mathrm{~h}$ dehydration treatment and recovered to normal levels at 24 h. PbrPUB1, PbrPUB38 and PbrPUB25, exhibited highest expression level at $1 \mathrm{~h}$, where PbrPUB12, PbrPUB14, PbrPUB3 and PbrPUB36 exhibited highest expression level at $12 \mathrm{~h}$ under dehydration treatment. These results suggested that $P b r P U B 1, P b r P U B 38$ and $P b r P U B 25$ respond to dehydration treatment faster than that of PbrPUB12, PbrPUB14, PbrPUB3 and PbrPUB36. Therefore, PUB gene family in pear play vital role in the process of low temperature stress response. 
To verify whether PUB gene in pear involve in low temperature stress, the seedlings were placed in the chamber set at $4^{\circ} \mathrm{C}$ for $0,1,6,9,12,24,48$ and $96 \mathrm{~h}$ (Fig. 6b). We detected the expression level of those 11 PbrPUB genes by qRT-PCR experiment. 4 genes (PbrPUB12, PbrPUB3, PbrPUB36 and PbrPUB48) were up-regulated expressed under cold stress, suggesting that those $P b r P U B$ genes might respond to low temperature. PbrPUB12, PbrPUB48 and PbrPUB36 were highly increased during the $48 \mathrm{~h}$ low temperature exposure. The expression level of $P b r P U B 3$ was reached to double peak at $1 \mathrm{~h}$ and $48 \mathrm{~h}$.

To explore the functions of $P b r P U B$ genes under the salt stress, the seedlings were placed in solution containing $200 \mathrm{mM} \mathrm{NaCl}$ solution for $0,2,4,6,8,12$ and $36 \mathrm{~h}$ (Fig. 6c). We detected the expression level of those 11 PbrPUB genes by qRT-PCR experiment. On the whole, all of the selected 11 PbrPUB genes were significantly up-regulated expressed under the $200 \mathrm{mM}$ salt stress treatment. The expression level of PbrPUB14, PbrPUB25, PbrPUB3, PbrPUB48 and PbrPUB7 were highly increased during the $12 \mathrm{~h}$ salt exposure. Moreover, PbrPUB1, PbrPUB12, PbrPUB18, PbrPUB34, PbrPUB36 and PbrPUB38 were highest expressed at $4 \mathrm{~h}$ under salt stress, suggesting that these 6 PbrPUB genes respond to salt treatment actively. We focus on the expression level of PbrPUB18. In the 4-8 h, the expression level of PbrPUB18 was significantly increased, and then it was down-regulated at $12 \mathrm{~h}$, finally recovered normal level at $36 \mathrm{~h}$.

Previous study had reported that PUB gene involved in ABA-mediated drought stress responses. To investigate the roles of PUB gene family in $A B A$ stress, the seedlings were dipped in solution containing $100 \mu \mathrm{M}$ ABA for $0,1,3,6,9,12$ and $36 \mathrm{~h}$ (Fig. 6d). As results, all of 11 PUB genes were respond to the ABA stress, and these gene were unregulated expressed at first, and then were down-regulated at $36 \mathrm{~h}$ after $A B A$ treatment. These results indicated that PUB genes play important roles in ABA-regulated pathway. The expression levels of three genes (PbrPUB1, PbrPUB25, and PbrPUB36) were reached to peak at $1 \mathrm{~h}$, suggesting that these three genes were actively responded to $A B A$ stress. Interestingly, we found that PbrPUB18 was expressed in $6 \mathrm{~h}$ and $12 \mathrm{~h}$ after ABA treatment.

\section{Subcellular localization of PbrPUB18}

To further explore the function of PUB gene in pear, we constructed subcellular localization experiment to identify where the PUB genes act function. To further verify the biologic function of $P b r P U B$ genes in pear under drought stress, PbrPUB18 was selected from 9 up-regulated expressed genes for further study. The full-length ORF of PbrPUB18 was fused to the N-terminal of GFP (Green Fluorescent Protein), under the control of CaMV35S promoter to form a fusion construct 35S-PbrPUB18-GFP. Then the 35S-PbrPUB18GFP fusion protein and 35S-GFP (as a control) was transient transformed into tobacco leaves and the fluorescence signal was observed with a confocal laser scanning microscope. The green fluorescence of GFP control was found in the membrane and the nucleus (Fig. 7a). In contrast, 35S-PbrPUB18-GFPfused GFP protein was only existed in the nucleus and integrated perfectly with DAPI (4', 6-diamidino-2phenylindole) regime (Fig. 7b), suggesting that PbrPUB18 was located in the nucleus, which was consistent with our prediction in Table 1.

Assessment of drought tolerance in transgenic lines of PbrPUB18 
To further confirm the biologic function of PbrPUB18 gene under drought stress, Arabidopsis Col-0 plants (WT) were transformed by the floral dip method [37]. Two overexpression lines OE-4 and OE-5 were screened out by PCR identification at DNA level in T0 generation plants. The selected robust Arabidopsis thaliana seedlings were transplanted into the soil as $\mathrm{T} 1$ generation plants, and the $\mathrm{T} 1$ generation positive seedlings were identified again by semi-quantitative PCR at mRNA level. QRT-PCR also verified the expression of PbrPUB18 in OE-4 and OE-5 far above in WT (Additional file 3 Fig S3). To assess the function of overexpression PbrPUB18 in Arabidopsis on drought tolerance, 15-day-old WT and transgenic lines were used to expose to drought stress by withholding water for 12 days. There was no morphological difference between WTs and the transgenic lines in the normal condition. After 12 days without water, the two transgenic lines showed more tolerance to the drought stress, as manifested by lesser leaf-wilting symptoms compared with the WT plants (Fig. 8a). In addition, chlorophyll fluorescence measurements were recorded to further verify drought tolerance of WTs and the transgenic lines (Fig. 8b). The maximum quantum efficiency of the photochemistry (Fv/Fm) values was not affected by species and growth conditions, but under stress conditions, this parameter decreased significantly. After 12 days drought treatment, the Fv/Fm values of WT was significantly lower than of the two transgenic lines, suggesting WT showed more sensitivity to the drought stress (Fig. 8e). Electrolyte leakage (EL), an important indicator of cell injury, was measured after drought stress. The EL of two transgenic lines were only approximate $15 \%-20 \%$ compared to WT (37.3\%), suggesting that WT suffered more severe membrane damage than transgenic lines of Arabidopsis by overexpressing PbrPUB18 (Fig. 8c). The transgenic plants displayed significantly lower malondialdehyde (MDA) contents than WT exposure to drought condition (Fig. 8d).

In the drought tolerance stress assay, we found that two transgenic lines had lower values of EL and MDA, implying that they might be subjected to lighter extent oxidative stress than the wild type. Histochemical staining with 3, 3'-diaminobenzidine (DAB) and nitro-blue tetrazolium chloride (NBT) was used to analyze the in situ accumulation of $\mathrm{H}_{2} \mathrm{O}_{2}$ and $\mathrm{O}_{2}$, two main reactive oxygen species (ROS), respectively. After drought stress the leaves of WT lines (Fig. 8f) were stained to a more serious extent compared with transgenic Arabidopsis, implying that more ROS was produced in the WT under the drought conditions. Similar to staining results, quantitative measurements further demonstrated that $\mathrm{H}_{2} \mathrm{O}_{2}$ contents in the two transgenic lines were remarkably lower than those of WT (Fig. 8g). And anti- $\mathrm{O}_{2}{ }^{-}$ contents in the two transgenic lines were remarkably more than those of WT (Fig. 8h), implying that less ROS was produced in the two transgenic lines under the drought conditions.

\section{Discussion}

\section{Genome-wide and phylogenetic analysis of PbrPUB genes in pear}

As a family of ubiquitin ligases, U-box genes encode a conserved U-box motif of about 70 amino acids and regulated the ubiquitination of the substrates [23]. U-box genes were widely distributed in the plants and reported to participate in many biological processes including plant hormone signaling regulations [6], self-incompatible or pseudo-self-compatibility regulations [18] as well as in biotic stress [19-21] and 
abiotic stress $[5,22,23]$. Due to PUB gene play important role during plant development, PUB genes have been identified in different plant species, such as Arabidopsis thaliana $(61)[9,10]$, rice (77) [11], tomato (62) [12], cotton (93) [13], and banana (91) [14]. Pear, one of Rosaceae fruit trees, is widely cultivated all over the world. However, the analysis related to PUB genes in pear was poor until now. In the present study, 62 genes were identified as PUB gene family in pear using bioinformatics analysis, and the number of PUB gene in pear is similar to that of Arabidopsis thaliana (61) and tomato (62). We therefore speculate that the number of PUB genes in plant kingdom is relatively conserved.

Phylogenetic tree analysis indicated that a total of 185 PUB protein members in these three species (containing 62 pear, 62 tomato, and 61 Arabidopsis) were categorized into five subgroups (I-V). Although there are some differences, this phylogenetic tree was largely consistent with the results from a previous study $[12,14]$. For example, $125 \mathrm{GmPUB}$ genes in soybean proteins were classified into six groups using phylogenetic tree analysis [17]. Through the phylogenetic relationship analysis, it was showed that PbrPUBs exhibited closer relations with SIU-boxs compared with AtPUBs. This result was consistent with the fact that pear and tomato exhibited closely relationship than Arabidopsis. Although the number of PUB genes was similar in three species, we found that the genes of Group I in had undergone rapid expansion and Group II had undergone rapid lost. In addition to the U-box domain, 62 PbrPUB proteins are found to bind to different domains including armadillo (ARM) repeats, the tetratricopeptide (TPR) domain and WD40 repeats. The majority of PUB proteins that have been elucidated for biological functions are from the U-box proteins with ARM repeats [18]. The ARM repeats have been shown primarily mediating the interaction with substrates, suggesting that interaction make the substrates available for ubiquitination [23]. 25 member of PUB genes in pear only housed U-box domain, and 25 members housed both U-box and ARM domain. Moreover, TPR domain was found in PbrPUB14 gene and WD40 repeats was found in PbrPUB40 gene.

\section{The function predication of PUB gene family in pear based on cis-acting and specific-tissues expression analysis}

The cis-acting analysis of putative promoter indicated the U-box gene family was involved in stressrelated mechanisms, hormonal regulation, growth and development. Previous study had reported that PUB proteins were responded with ABA. For instance, AtPUB44 could regulated the biosynthesis of ABA through ubiquitinating the $\mathrm{AAO} 3$ (abscisic aldehyde oxidase 3) via 26 proteasomes [38]. In additional, one transcription factor of $A B \mid 3$ was regulated by AtPUB 9 and increased the ABA sensitivity of Arabidopsis during seedling germination [39]. AtPUB18, AtPUB19 and AtPUB44 were found to directly interrupt the biosynthesis of $A B A$ directly. In our study, 55 genes contained the $A B A$ responsiveness elements on the putative promoter region. Especially, we found that eight $A B A$ responsiveness elements were identified in the promoter region of $P b r P U B 43$. This result indicted that PUB gene might play important role during ABA signal transduction in pear. In Arabidopsis and Nicotiana, the expression levels of PUB genes were regulated by abiotic and biotic stress [40]. In here, we found MYB binding site involved in drought induction responsive element, abscisic acid responsive element, defense and stress responsive element, low temperature responsive element, wound responsive element in the promoter region of PUB genes in

Page 10/29 
pear. The present of these elements indicated that most of PUB genes in pear were involved in stress response.

Based on our previous RNA-Seq data in seven different tissues of 'Dangshansuli' cultivar pear, transcriptome sequencing analysis and qRT-PCR expression profiling were conducted to investigate PUB gene expression patterns in seven different tissues. The members of the PUB gene family exhibited a variety of expression patterns in seven different tissues. These results indicated that PUB might particular in a diversity functions during plant growth and development. Among the 62 members of PUB gene family in pear, 29 PbrPUB genes were expressed in all seven different tissues. Additionally, $72.58 \%$ of PbrPUBs were detected in transcriptional abundance in pear sepal. Whereas of PbrPUBs expressed in all tissues, $45.16 \%$ were highest in leaves, suggesting these genes may have important roles in the development of pear leaves. Further, the expression levels of 15 randomly selected members of the PbrPUB gene family were validated using qRT-PCR. Based on qRT-PCR expression profiles, we found that

the 15 PbrPUB genes were highly expressed in petal, sepal, ovary and leaf, suggesting that $P b r P U B$ genes may function in the development of petal, sepal, ovary and leaf.

\section{Roles of PbrPUB genes in response to different abiotic stresses}

Previous studies have reported that PUB genes in plants involved in the process of stress responses [24, $26,41,42]$. A large of PUB genes were induced expressed during abiotic stress conditions [40]. In this study, the differential expression levels of 11 PbrPUB genes under various abiotic stresses were investigated by using qRT-PCR, including drought, low temperature and salt stress. From the result, PbrPUB12, PbrPUB3, PbrPUB36 and PbrPUB48 were significantly up-regulated expressed under four treatment, suggesting these three genes could response to dehydration, $A B A$, cold and salt stress. PbrPUB7 was down-regulated expressed under dehydration stress, suggesting that $P b r P U B 7$ might negatively regulate the response process of dehydration. Drought is one of most critical stresses and could significantly affect the growth of plant. In our study, we found that the expression level of PbrPUB18 were significant up-regulated after dehydration treatment, suggesting that $P b r P U B 18$ genes might respond to drought stress. Therefore, $P b r P U B 18$ gene was selected for further functional identification. Subcellular localization experiment suggested that PbrPUB18 was located the cell nucleus. This result indicated that $P b r P U B 18$ might act biology function at the cell nucleus. Furthermore, different from previous studies, overexpression of $P b r P U B 18$ gene in Arabidopsis thaliana resulted in resistance to drought tolerance. Summary, we systematically identified the PUB gene family in pear, and further function identification laid a foundation for the functional study of PUB genes of in pear in future.

\section{Conclusions}

In our study, a total of 62 PbrPUB members were identified in Chinese white pear genome, and were unevenly distributed on 17 pear chromosomes. According to phylogenetic tree analysis, the pear PUB gene family was divided into five groups. The conserved motif and gene structure analysis provided strong evidence to support the result of classification phylogenetic tree. Cis-acting element analysis 
indicted $P U B$ genes might participate in diverse biological processes, especially in the response to abiotic stresses and phytohormones. Transcription sequencing data from different seven tissues exhibited diverse of expression level of $P b r P U B$ genes. Further qRT-PCR was used to identify candidate genes associated with abiotic stresses. In addition, PbrPUB18 was cloned and functionally identified. Subcellular localization revealed $P b r P U B 18$ genes were located on cell nucleus. Heterologous overexpression of PbrPUB18 in Arabidopsis indicated that the over-expression of PbrPUB18 could enhance resistance in drought treatment. Our data and analysis lay a solid foundation for the future research on molecular mechanism of PbrPUB genes in responding to abiotic stresses.

\section{Methods}

\section{Genome identification of $P U B$ gene family members in Chinese white pear}

To identify the potential members of the PUB protein family, the complete genome sequence of pear (Pyrus bretschneideri) was downloaded from the pear genome project (http://peargenome.njau.edu.cn/) [32]. The hidden Markov Model (HMM) profile of the U-box domain (PF04564) was obtained from Pfam30.0 (http://pfam.xfam.org/), and was used as a query to identify the candidate PUBs from the pear genome protein database using HMMER3.0.

All candidate PUB genes were further verified by using SMART conserved domain search tools (http://smart.embl-heidelberg.de/) to ensure that each candidate protein contained a U-box domain. In addition, the ExPASy ProtParam (https://www.expasy.org/), an online proteomics and sequence analysis tool, was performed to predict the physical and chemical properties of PbrPUB proteins, such as the number of amino acids (AA), molecular weights, isoelectric points, and instability index [43]. The subcellular localization prediction was carried out in Cell-PLoc 2.0 (http://www.csbio.sjtu.edu.cn/bioinf/Cell-PLoc-2/) [44].

\section{PbrPUB protein phylogenetic analysis}

Arabidopsis PUB protein sequences were downloaded from the Arabidopsis Information Resource (TAIR) (https://www.Arabidopsis.org/browse/genefamily/pub.jsp) [10] Tomato PUB protein sequences were obtained from previous study [12]. All PUB full-length protein sequences in Pyrus bretschneideri, Arabidopsis thaliana and Solanum lycopersicum were aligned using ClustalW withed fault parameters. Then, MEGA-X was used to construct an unrooted phylogenetictree based on the result of multiple sequence alignment using the $\mathrm{NJ}$ (Neighbor Joining) method with 1000 bootstrap replicates [45]. Evolview (https://evolgenius.info//evolview-v2/\#login) [46] was used to visualize the phylogenetic tree.

Gene structure, motif analysis and cis-acting elements analysis

To identify and visualize the structural organization (introns, exons, and untranslated regions) of the pear PUB gene family, the information of gene structure was extracted from whole genome database of pear 
using in-house scripts. The novel conserved motifs of PbrPUB genes were identified by MEME suite (http://meme-suite.org/tools/meme). A total of 20 motifs and a width limit of 200 amino acids were used for the analysis with other default parameters. TBtools were used to visualize the results of gene structure and conserved motif analysis.

Bedtools software was used to extract the $2000 \mathrm{bp}$ upstream (putative promoter region) sequences of the transcription start site of all PbrPUB genes [47]. Then, PlantCare

(http://bioinformatics.psb.ugent.be/webtools/plantcare/html/) was used to predict the cis-acting elements in the putative promoter region of $P b r P U B$ genes [48]. TBtools were used to visualize the distribution of putative cis-acting elements.

\section{Synteny analysis and chromosomal localization}

Synteny analysis was performed by the method described in the Plant Duplicate Gene Database (PlantDGD) (http://pdgd.njau.edu.cn:8080/). The collinear block was identified by PbrPUB duplication events in the MCScanX [49]. The data were integrated and plotted by using Circos [50]. Based on the annotation information of PUB genes in pear, the corresponding location distributions of PbrPUB genes in chromosomes were displayed by TBtools [51].

\section{Gene expression analysis of PbrPUB on the RNA-SEq. Data}

The RNA-seq data of the cultivar 'Dangshansuli' in seven different tissues were acquired from our previous study, including bud, stem, leaf, sepal, petal, fruit and ovary [52]. We downloaded them from the National Center for Biotechnology Information database (NCBI, https://www.ncbi.nlm.nih.gov/). Then, Heatmap.2, afunction in $\mathrm{R}$, was used to plot heatmaps based on thelog $2(R P K M+1)$ value of each PbrPUB gene.

\section{Plant materials and stress treatments}

The test material Pyrus betulaefolia were collected from the pear germplasm orchard of the Center of Pear Engineering Technology Research situated at Hushu in Nanjing, which were cultivated in the experimental base of the National Center of Pear Engineering Technology Research, Nanjing Agricultural University. To explore the expression levels of $P b r P U B$ genes under abiotic Stresses, uniform and healthy shoots of Pyrus betulaefolia from grown 45-day-old seedlings were subjected to various stress treatments. The shoots were washed and cultured for 1 day in a growth chamber to minimize the mechanical stress on the tissues, followed by exposure to corresponding stress treatments, which were carried out as follows. For dehydration treatment, the shoots were placed on dry filter papers for $0,1,6,9$, 12 and $24 \mathrm{~h}$ at ambient environment. For cold stress, the seedlings were placed in the chamber set at $4^{\circ} \mathrm{C}$ for $0,1,6,9,12,24,48$ and $96 \mathrm{~h}$. For salt stress, the seedlings were placed in solution containing $200 \mathrm{mM}$ $\mathrm{NaCl}$ solution for $0,2,4,6,8,12$ and $36 \mathrm{~h}$. In addition, ABA treatment was carried out as follows. The seedlings were dipped in solution containing $100 \mu \mathrm{M}$ ABA for $0,1,3,6,9,12$ and $36 \mathrm{~h}$. For each treatment, at least 30 seedlings were used, and the leaves were sampled from three randomly collected seedlings at 
the designated time points, immediately frozen in liquid nitrogen and stored at $-80^{\circ} \mathrm{C}$ for the purpose of extracting RNA for expression analysis.

\section{Quantitative real-time PCR (qRT-PCR) analysis}

Total RNA was isolated using a Plant Total RNA Isolation Kit Plus(FOREGENE Co. Ltd., Chengdu, China) and reverse transcribed into CDNA using the PrimeScript ${ }^{\mathrm{TM}} \mathrm{RT}$ reagent kit (Takara, Dalian, China) according to manufacturer's the instructions. QRT-PCR was performed on a BioRad CFX96 real-time system using an SYBR ${ }^{\circledR}$ Green PCR Master Mix kit (Takara) using $20 \mu \mathrm{L}$ of reaction mixture consisting 10 $\mu \mathrm{L}$ of $2 \subseteq$ SYBR-PreMix EX Taq, $0.25 \mu \mathrm{M}$ of each primer (Additional file 4: Table S1) and $50 \mathrm{ng}$ of cDNA template. Fifteen pairs of the most specific primers were designed by Primer 5.0 software (PREMIER Biosoft International, Palo Alto, CA, USA) and checked by using NCBI online software (https://www.ncbi.nlm.nih.gov/). The protocol for real-time PCR was as follows: initiation with a 10 min denaturation at $95^{\circ} \mathrm{C}$, followed by 55 cycles of amplification with $15 \mathrm{~s}$ of denaturation at $95^{\circ} \mathrm{C}, 15 \mathrm{~s}$ of annealing at $58^{\circ} \mathrm{C}$ and $20 \mathrm{~s}$ of extension at $72^{\circ} \mathrm{C}$. Reads for fluorescence data collection occurred at $60^{\circ} \mathrm{C}$. A melting curve was performed from 60 to $95^{\circ} \mathrm{C}$ to check the specificity of the amplified product.

The $2^{-\Delta \Delta C T}$ method was applied to calculate the relative expression level of each gene [53]. Relative expression levels were calculated by normalizing to expression of the pear Tubulin gene (AB239681), which was used as an internal control for Pyrus betulaefolia. Expression of AtActin was used as an internal reference for Arabidopsis. The expression analysis at each time point was repeated at least three times, and the data are shown as the mean values \pm SE.

\section{Subcellular localization}

The full-length cDNA of PbrPUB18 without a stop codon was amplified by RT-PCR with primer pair (GSP16, Additional file 4: Table S1) containing restriction sites of $\mathrm{Xba}$ I and $\mathrm{BamHI}$, then inserted into the pCAMBIA1302 vector and fused in-frame to the N-terminal of GFP (Green Fluorescent Protein), under the control of CaMV35S promoter to form a fusion construct 35S pro: PbrPUB18-GFP. After validation by sequencing, the fusion constructs 35S pro: PbrPUB18-GFP and 35S pro: GFP (as a control) were mobilized into Agrobacterium tumefaciens strain GV3101 by heat shock. Transient transformation of Nicotiana benthamiana was done as described earlier [54]. The fluorescence signal was observed with a confocal laser scanning microscope (LSM410; Carl Zeiss) after $72 \mathrm{~h}$ post infiltration and the position of nucleus was revealed by staining with 4', 6-diamidino-2-phenylindole (DAPI).

\section{Arabidopsis transformation and characterization of transgenic plants}

Arabidopsis thaliana ecotype Columbia Col-0 plants were transformed for heterologous over-expression PbrPUB18 by using the floral dip method [37]. And Agrobacterium tumefaciens suspension containing the vector 35S-PbrPUB18-GFP $\left(\mathrm{OD}_{600}=0.80\right)$ was used for transformation. T0 seeds were identified by Murashige and Skoog (MS) solid mediumwith $20 \mathrm{mg} \cdot \mathrm{L}^{-1}$ hygromycin and then verified by PCR analysis using specific primers pair (GSP17, Additional file 4: Table S1). Semi-quantitative RT-PCR and qRT-PCR 
was used to further analyze the transcript levels of PbrPUB18 in T1 plants with primers pair (GSP18 and GSP5, Table S1). Two overexpressing lines (OE-4 and OE-5) of PbrPUB18 were selected to generate T3 homozygous plants for the subsequent stress tolerance assay.

\section{Assessment of drought tolerance in transgenic lines}

To test the drought tolerance, The WT and transgenic lines were subjected to drought. For drought treatments, 15-day-old soil-grown Arabidopsis seedlings were subjected to withholding water for 12 days. At the end of treatment, the leaves were collected for measurement of electrolyte leakage, MDA content and ROS level. Electrolyte leakage was measured as described [55]. The MDA content, $\mathrm{H}_{2} \mathrm{O}_{2}$ and $\mathrm{O}_{2}{ }^{-}$ content were measured using specific analytical kits (Nanjing Jiancheng Bioengineering Institute, Jiangsu, China) following the manufacturer's instructions. Histochemical staining with 3, 3'diaminobenzidine (DAB) and nitro-blue tetrazolium chloride (NBT) was used to analyse the in situ accumulation of $\mathrm{H}_{2} \mathrm{O}_{2}$ and $\mathrm{O}_{2}{ }^{-}$respectively, according to [56]. In addition, chlorophyll fluorescence measurements were recorded using an IMAGING-PAM chlorophyll fluorometer and ImagingWin software (Walz; Effeltrich, Germany) according to [57]. For chlorophyll fluorescence imaging, the 20 mins-darkadapted plants were illuminated under an initial saturating pulse of $>1800 \mu \mathrm{mol}$ photons $\mathrm{m}^{-2} \mathrm{~s}^{-1}$. Then the maximum quantum efficiency of the photochemistry (Fv/Fm) values was obtained.

\section{Statistical analysis}

Three independent technical replicates were used for each sample, shown as mean \pm standard error (SE). Statistical analyses were carried out with SPSS (IBM SPSS 17) statistical software package. Analysis of variance (ANOVA) was used to compare the statistical difference based on Duncan's multiple range test, at the significance levels of $\mathrm{P}<0.05(*), \mathrm{P}<0.01(* *)$, and $\mathrm{P}<0.001(* * *)$.

\section{Abbreviations}

PUB: Plant U-box gene; UPS: Ubiquitin/26S proteasome system; Ub: Ubiquitin; E1: Ubiquitin-activating enzyme; E2: Ubiquitin- conjugating enzyme; E3: Ubiquitin ligase; HECT: Homology to E6-Associated Carboxy-Terminus; RING: Really Interesting New Gene; CRLs: Cullin-RING ligases; UFD2: Ubiquitin fusion degradation protein-2; AAO3: Abscisic aldehyde oxidase 3; ABA: Abscisic acid; MeJA: Methyl jasmonate; GAs: Gibberellin; RPKM: Reads per kilobase per million; qRT-PCR: Quantitative real-time PCR; GFP: Green fluorescent protein; DAPI: 4', 6-diamidino-2-phenylindole; Fv/Fm: The maximum quantum efficiency of the photochemistry; WT: Wide type; MDA: Malondialdehyde; EL: Electrolyte leakage; DAB: 3, 3'diaminobenzidine; NBT: Nitro-blue tetrazolium chloride; ROS: Reactive oxygen species; TPR: Tetratricopeptide; ARM: Armadillo

\section{Declarations}

\section{Ethics approval and consent to participate}

Not Applicable. 


\section{Consent for publication}

Not Applicable.

\section{Availability of data and material}

All needed genome sequences and genome annotation files of Chinese white pear were obtained from the Nanjing Agricultural University pear genome project website (http://peargenome.njau.edu.cn). All data generated or analysed during this study are included in this published article and its supplementary information files

\section{Competing interests}

The authors declare that they have no competing interests.

\section{Funding}

This work has been supported by the National Key Research and Development Program of China (2019YFD1000102), the National Science Foundation of China (31872070; 32072538), the Jiangsu Agriculture Science and Technology Innovation Fund (CX(18)3065),the Fundamental Research Funds for the Central Universities of Nanjing Agricultural University (KYZ201607), the College of Horticulture SRT project of the Nanjing Agriculture University (202011YX05), and the Undergraduate Training Program for Innovation and Entrepreneurship (S20190040).

\section{Authors' contributions}

CMW and XSH designed and carried out the experiments, and CMW carried out all bioinformatics analysis and wrote the manuscript. YQD and BBS contributed to genes expression analysis. BBS and XSH directed and revised the manuscript. All authors read, reviewed and approved the final manuscript.

\section{Acknowledgements}

Not Applicable.

\section{References}

1. Gong Z, Xiong L, Shi H, Yang S, Herrera-Estrella LR, Xu G, Chao DY, Li J, Wang PY, Qin F et al: Plant abiotic stress response and nutrient use efficiency. Sci China Life Sci 2020, 63(5):635-674.

2. Hirayama T, Shinozaki K: Research on plant abiotic stress responses in the post-genome era: past, present and future. Plant $J$ 2010, 61(6):1041-1052.

3. McClellan AJ, Tam S, Kaganovich D, Frydman J: Protein quality control: chaperones culling corrupt conformations. Nat Cell Biol 2005, 7(8):736-741. 
4. Vierstra RD: The ubiquitin-26S proteasome system at the nexus of plant biology. Nat Rev Mol Cell Biol 2009, 10(6):385-397.

5. Zhang ZY, Li JH, Liu HH, Chong K, Xu YY: Roles of ubiquitination-mediated protein degradation in plant responses to abiotic stresses. Environ Exp Bot2015, 114:92-103.

6. Santner A, Estelle M: The ubiquitin-proteasome system regulates plant hormone signaling. Plant $J$ 2010, 61(6):1029-1040.

7. Jansen AH, Reits EA, Hol EM: The ubiquitin proteasome system in glia and its role in neurodegenerative diseases. Front Mol Neurosci 2014, 7:73.

8. Schulman BA, Harper JW: Ubiquitin-like protein activation by E1 enzymes: the apex for downstream signalling pathways. Nat Rev Mol Cell Biol2009, 10(5):319-331.

9. Azevedo C, Santos-Rosa MJ, Shirasu K: The U-box protein family in plants. Trends Plant Sci 2001, 6(8):354-358.

10. Wiborg J, O'Shea C, Skriver K: Biochemical function of typical and variant Arabidopsis thaliana U-box E3 ubiquitin-protein ligases. Biochem J 2008, 413(3):447-457.

11. Zeng LR, Park CH, Venu RC, Gough J, Wang GL: Classification, expression pattern, and E3 ligase activity assay of rice U-box-containing proteins. Mol Plant 2008, 1(5):800-815.

12. Sharma B, Taganna J: Genome-wide analysis of the U-box E3 ubiquitin ligase enzyme gene family in tomato. Sci Rep 2020, 10(1):9581.

13. Lu X, Shu N, Wang D, Wang J, Chen X, Zhang B, Wang S, Guo L, Chen C, Ye W: Genome-wide identification and expression analysis of PUB genes in cotton. BMC Genomics 2020, 21(1):213.

14. Hu H, Dong C, Sun D, Hu Y, Xie J: Genome-Wide Identification and Analysis of U-Box E3 Ubiquitin(-)Protein Ligase Gene Family in Banana. Int J Mol Sci 2018, 19(12).

15. Song J, Mo X, Yang H, Yue L, Song J, Mo B: The U-box family genes in Medicago truncatula: Key elements in response to salt, cold, and drought stresses. PLoS One 2017, 12(8):e0182402.

16. Wang C, Duan W, Riquicho AR, Jing Z, Liu T, Hou X, Li Y: Genome-wide survey and expression analysis of the PUB family in Chinese cabbage (Brassica rapa ssp. pekinesis). Mol Genet Genomics 2015, 290(6):2241-2260.

17. Wang N, Liu Y, Cong Y, Wang T, Zhong X, Yang S, Li Y, Gai J: Genome-Wide Identification of Soybean U-Box E3 Ubiquitin Ligases and Roles of GmPUB8 in Negative Regulation of Drought Stress Response in Arabidopsis. Plant Cell Physio/ 2016, 57(6):1189-1209.

18. Yee D, Goring DR: The diversity of plant U-box E3 ubiquitin ligases: from upstream activators to downstream target substrates. J Exp Bot 2009, 60(4):1109-1121.

19. Zeng LR, Qu S, Bordeos A, Yang C, Baraoidan M, Yan H, Xie Q, Nahm BH, Leung H, Wang GL: Spotted leaf11, a negative regulator of plant cell death and defense, encodes a U-box/armadillo repeat protein endowed with E3 ubiquitin ligase activity. Plant Cell 2004, 16(10):2795-2808.

20. Yang CW, Gonzalez-Lamothe R, Ewan RA, Rowland O, Yoshioka H, Shenton M, Ye H, O'Donnell E, Jones JD, Sadanandom A: The E3 ubiquitin ligase activity of arabidopsis PLANT U-BOX17 and its 
functional tobacco homolog ACRE276 are required for cell death and defense. Plant Cell 2006, 18(4):1084-1098.

21. Orosa B, He Q, Mesmar J, Gilroy EM, McLellan H, Yang C, Craig A, Bailey M, Zhang C, Moore JD et al: BTB-BACK Domain Protein POB1 Suppresses Immune Cell Death by Targeting Ubiquitin E3 ligase PUB17 for Degradation. PLoS Genet 2017, 13(1):e1006540.

22. Kai S, Yang W: E3 Ubiquitin Ligases: Ubiquitous Actors in Plant Development and Abiotic Stress Responses. Plant Cell Physio/2017(9):1461.

23. Trujillo M: News from the PUB: plant U-box type E3 ubiquitin ligases. J Exp Bot 2018, 69(3):371-384.

24. Liu YC, Wu YR, Huang XH, Sun J, Xie Q: AtPUB19, a U-box E3 ubiquitin ligase, negatively regulates abscisic acid and drought responses in Arabidopsis thaliana. Mol Plant 2011, 4(6):938-946.

25. Dong HS, Ryu MY, Jammes F, Hwang JH, Turek M, Kang BG, Kim KWT: Roles of Four Arabidopsis UBox E3 Ubiquitin Ligases in Negative Regulation of Abscisic Acid-Mediated Drought Stress Responses. Plant Physio/2012, 160(1):556-568.

26. Wang X, Ding Y, Li Z, Shi Y, Wang J, Hua J, Gong Z, Zhou JM, Yang S: PUB25 and PUB26 Promote Plant Freezing Tolerance by Degrading the Cold Signaling Negative Regulator MYB15. Dev Cel/ 2019, 51(2):222-235 e225.

27. Sabine, Raab, Gabriele, Drechsel, Maryam, Zarepour, Wolfram, Hartung, Tomokazu, Koshiba: Identification of a novel E3 ubiquitin ligase that is required for suppression of premature senescence in Arabidopsis. Plant Journal 2009.

28. Adler G, Konrad Z, Zamir L, Mishra AK, Raveh D, Bar-Zvi D: The Arabidopsis paralogs, PUB46 and PUB48, encoding U-box E3 ubiquitin ligases, are essential for plant response to drought stress. $B M C$ Plant Biol 2017, 17(1):8.

29. Park JJ, Yi J, Yoon J, Cho LH, Ping J, Jeong HJ, Cho SK, Kim WT, An G: OsPUB15, an E3 ubiquitin ligase, functions to reduce cellular oxidative stress during seedling establishment. Plant $J 2011$, 65(2):194-205.

30. Han PL, Dong YH, Jiang H, Hu DG, Hao YJ: Molecular cloning and functional characterization of apple U-box E3 ubiquitin ligase gene MdPUB29 reveals its involvement in salt tolerance. $J$ Integr Agric2019, 18(7):1604-1612.

31. Huang X, Li K, Xu X, Yao Z, Jin C, Zhang S: Genome-wide analysis of WRKY transcription factors in white pear (Pyrus bretschneideri) reveals evolution and patterns under drought stress. $B M C$ Genomics 2015, 16(1):1104.

32. Wu J, Wang Z, Shi Z, Zhang S, Ming R, Zhu S, Khan MA, Tao S, Korban SS, Wang H et al: The genome of the pear (Pyrus bretschneideri Rehd.). Genome Res 2013, 23(2):396-408.

33. Wang N, Xing Y, Lou Q, Feng P, Liu S, Zhu M, Yin W, Fang S, Lin Y, Zhang T: Dwarf and short grain 1, encoding a putative U-box protein regulates cell division and elongation in rice. J Plant Physio/2017.

34. Sharma B, Taganna J: Genome-wide analysis of the U-box E3 ubiquitin ligase enzyme gene family in tomato. Sci Rep2020, 10(1):9581. 
35. Li Q, Qiao X, Yin H, Zhou Y, Dong H, Qi K, Li L, Zhang S: Unbiased subgenome evolution following a recent whole-genome duplication in pear (Pyrus bretschneideri Rehd.). Hortic Res2019, 6(1):34.

36. Stone SL: The role of ubiquitin and the $26 \mathrm{~S}$ proteasome in plant abiotic stress signaling. Front Plant 2014, 5(5):135.

37. Clough SJ, Bent AF: Floral dip: a simplified method for Agrobacterium-mediated transformation of Arabidopsis thaliana. Plant $J 1998,16(6): 735-743$.

38. Raab S, Drechsel G, Zarepour M, Hartung W, Koshiba T, Bittner F, Hoth S: Identification of a novel E3 ubiquitin ligase that is required for suppression of premature senescence in Arabidopsis. Plant $J$ 2009, 59(1):39-51.

39. Samuel MA, Mudgil Y, Salt JN, Delmas F, Ramachandran S, Chilelli A, Goring DR: Interactions between the S-domain receptor kinases and AtPUB-ARM E3 ubiquitin ligases suggest a conserved signaling pathway in Arabidopsis. Plant Physiol 2008, 147(4):2084-2095.

40. Yee D, Goring DR: The diversity of plant U-box E3 ubiquitin ligases: from upstream activators to downstream target substrates. J Exp Bot2009, 60(4):1109-1121.

41. Bergler J, Hoth S: Plant U-box armadillo repeat proteins AtPUB18 and AtPUB19 are involved in salt inhibition of germination in Arabidopsis. Plant Biol (Stuttg) 2011, 13(5):725-730.

42. Cho SK, Chung HS, Ryu MY, Park MJ, Lee MM, Bahk YY, Kim J, Pai HS, Kim WT: Heterologous expression and molecular and cellular characterization of CaPUB1 encoding a hot pepper U-Box E3 ubiquitin ligase homolog. Plant Physiol 2006, 142(4):1664-1682.

43. Panu A, Manohar J, Konstantin A, Delphine B, Gabor C, Edouard dC, Séverine D, Volker F, Arnaud F, Elisabeth G: ExPASy: SIB bioinformatics resource portal. Nucleic Acids Res2012(W1):W597.

44. Chou KC, Shen HB: Cell-PLoc: a package of Web servers for predicting subcellular localization of proteins in various organisms. Nat Protoc2007, 3(2):153-162.

45. Cummings MP: MEGA (Molecular Evolutionary Genetics Analysis): American Cancer Society; 2004.

46. Zhang H, Gao S, Lercher MJ, Hu S, Chen WH: EvolView, an online tool for visualizing, annotating and managing phylogenetic trees. Nucleic Acids Res 2012, 40(Web Server issue):W569-572.

47. Quinlan AR, Hall IM: BEDTools: a flexible suite of utilities for comparing genomic features. Bioinformatics 2010, 26(6):841-842.

48. Rombauts $S$, Dehais $P$, Van Montagu M, Rouze P: PlantCARE, a plant cis-acting regulatory element database. Nucleic Acids Res 1999, 27(1):295-296.

49. Wang Y, Tang H, Debarry JD, Tan X, Li J, Wang X, Lee TH, Jin H, Marler B, Guo H et al: MCScanX: a toolkit for detection and evolutionary analysis of gene synteny and collinearity. Nucleic Acids Res 2012, 40(7):e49.

50. Krzywinski M, Schein J, Birol I, Connors J, Gascoyne R, Horsman D, Jones SJ, Marra MA: Circos: an information aesthetic for comparative genomics. Genome Res 2009, 19(9):1639-1645.

51. Chen C, Chen H, Zhang Y, Thomas HR, Frank MH, He Y, Xia R: TBtools: An Integrative Toolkit Developed for Interactive Analyses of Big Biological Data. Mol Plant 2020, 13(8):1194-1202. 
52. Qionghou, Li, Xin, Qiao, Hao, Yin, Yuhang, Zhou, Huizhen, Dong: Unbiased subgenome evolution following a recent whole-genome duplication in pear (Pyrus bretschneideri Rehd.). Hortic Res 2019.

53. Livak KJ, Schmittgen TD: Analysis of Relative Gene Expression Data using Real-Time Quantitative PCR. Method 2002, 25(4):402-408.

54. Kumar KRR, Kirti PB: A mitogen-activated protein kinase, AhMPK6 from peanut localizes to the nucleus and also induces defense responses upon transient expression in tobacco. Plant Physiol Biochem2010, 48(6):481-486.

55. Dahro B, Wang F, Peng T, Liu JH: PtrA/NINV, an alkaline/neutral invertase gene of Poncirus trifoliata, confers enhanced tolerance to multiple abiotic stresses by modulating ROS levels and maintaining photosynthetic efficiency. BMC Plant Bio/2016, 16(1):76.

56. Huang XS, Liu JH, Chen XJ: Overexpression of PtrABF gene, a bZIP transcription factor isolated from Poncirus trifoliata, enhances dehydration and drought tolerance in tobacco via scavenging ROS and modulating expression of stress-responsive genes. BMC Plant Biol 2010, 10(1):230.

57. Woo NS, Badger MR, Pogson BJ: A rapid, non-invasive procedure for quantitative assessment of drought survival using chlorophyll fluorescence. Plant Methods 2008, 4(1):27.

\section{Tables}

Due to technical limitations, table 1 is only available as a download in the Supplemental Files section.

\section{Figures}




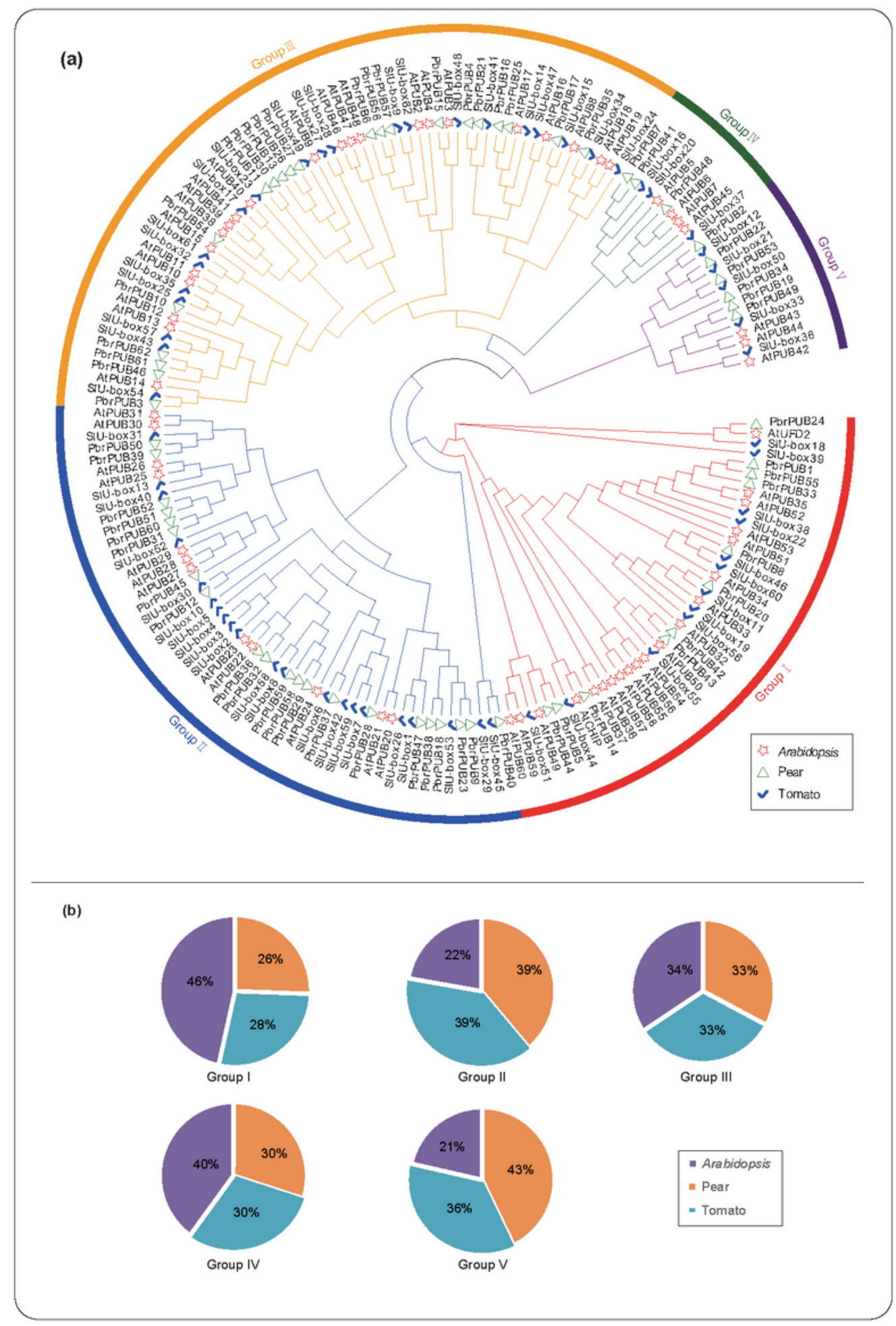

\section{Figure 1}

Phylogenetic tree analysis of PUB gene family. (a) A Neighbor-Joining ( $\mathrm{NJ}$ ) tree of PUB proteins from three species, including pear, tomato and Arabidopsis. The phylogenetic tree was constructed by Mega-X software with 1000 boot strap. The red star, green triangle and blue tick represents the PUB proteins in Arabidopsis, pear and tomato, respectively. All of 185 PUB genes from three species were clustered into five subgroups, named Group I, II, III, IV and V; (b) Five pie plots represented the percentage of PUB genes 
of three species in five groups. The orange part represented the percentage of PUB genes in pear, and the blue part represented the percentage of PUB genes in tomato, and the purple part presented the percentage of PUB genes in Arabidopsis.

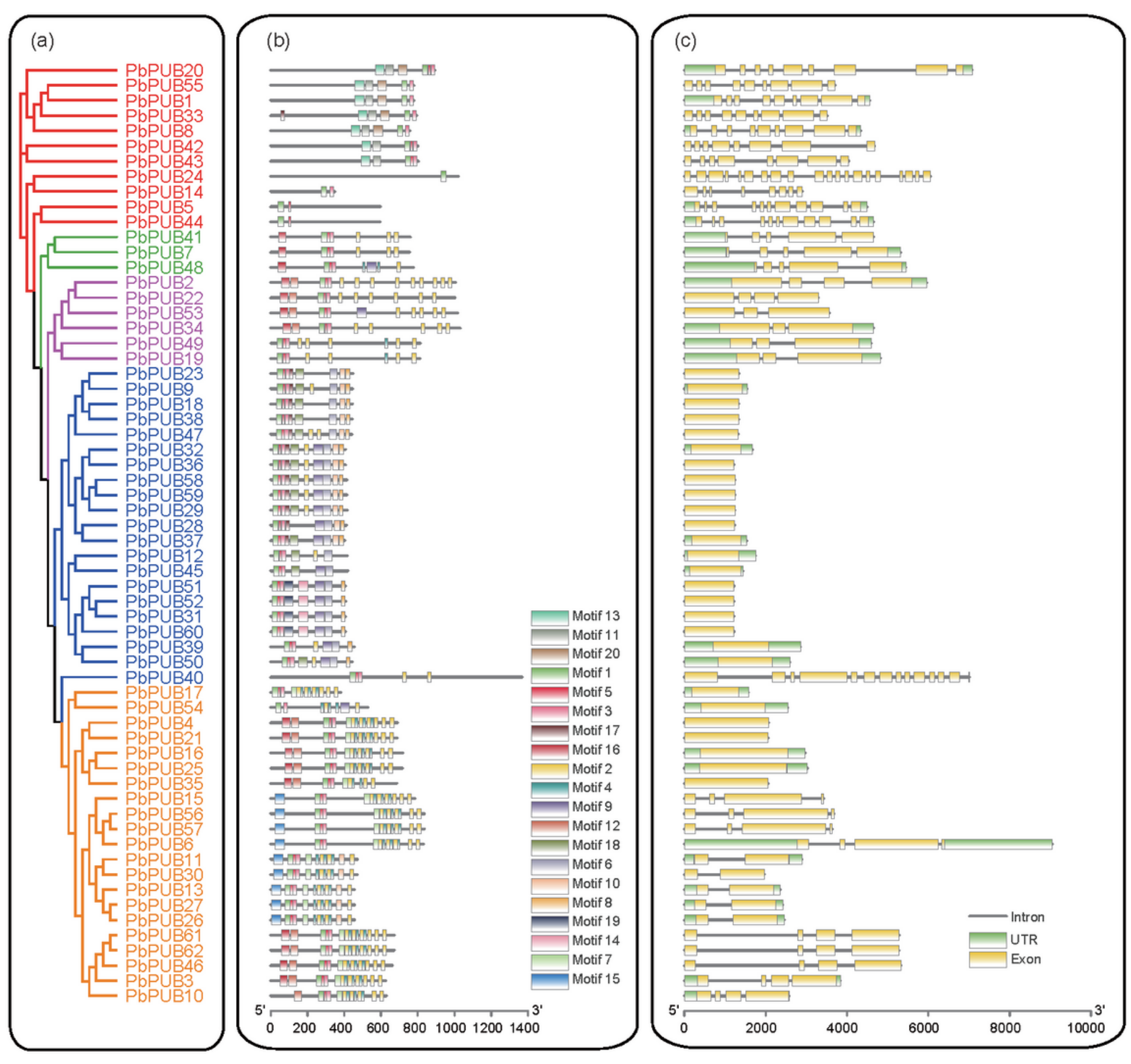

Figure 2

The conserved motifs and gene structure analysis of PUB gene family in pear. (a) A Neighbor-Joining $(\mathrm{NJ})$ phylogenetic tree of 62 pear PUB genes. The phylogenetic tree was constructed by Mega-X with 1000 bootstrap. The red branches indicated group I; the blue branches indicated group II; the orange branches indicated group III; the green branches indicated group IV; the purple branches indicated group V; (b) The conserved motifs analysis of PbrPUB genes in pear. A total of 20 motifs were predicated by 
MEME tool, named Motif 1-20. The scale bar indicates 200 aa; (c) The gene structure analysis of PUB genes in pear, including UTR, intron and exon. The green rectangles represented UTR; the yellow rectangles represented Exon; the grey lines presented Intron. The scale bar indicates $2 \mathrm{~kb}$.
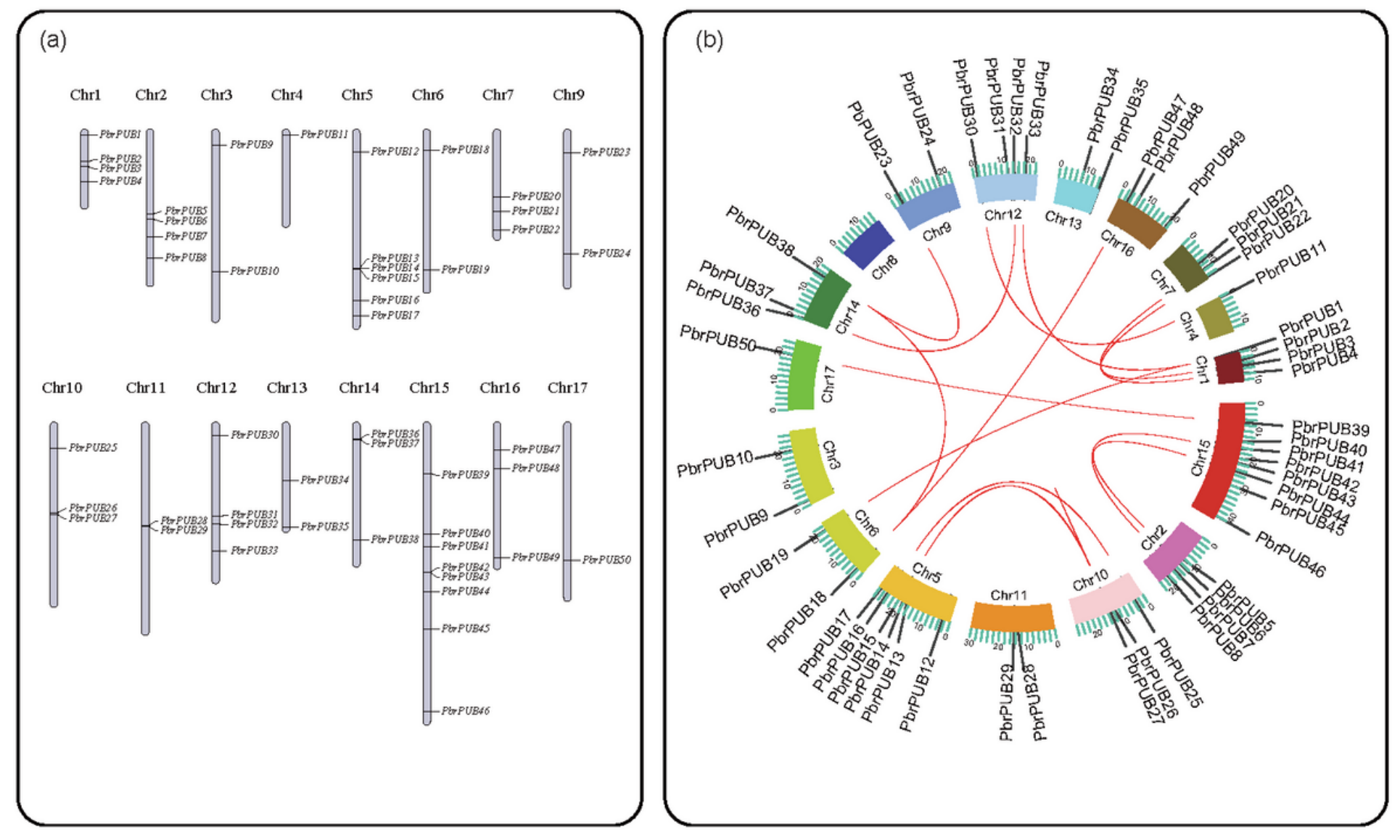

\section{Figure 3}

The location distribution and synteny analysis of PUB genes in pear genome. (a) The distribution pattern of PUB genes family in 17 pear chromosomes. Due to no PUB genes were mapped on Chromosomes 8 , we didn't show it in the Fig.; (b) The distribution pattern synteny analysis of PUB genes family. The red lines indicated the synteny gene pairs of PUB gene family. 

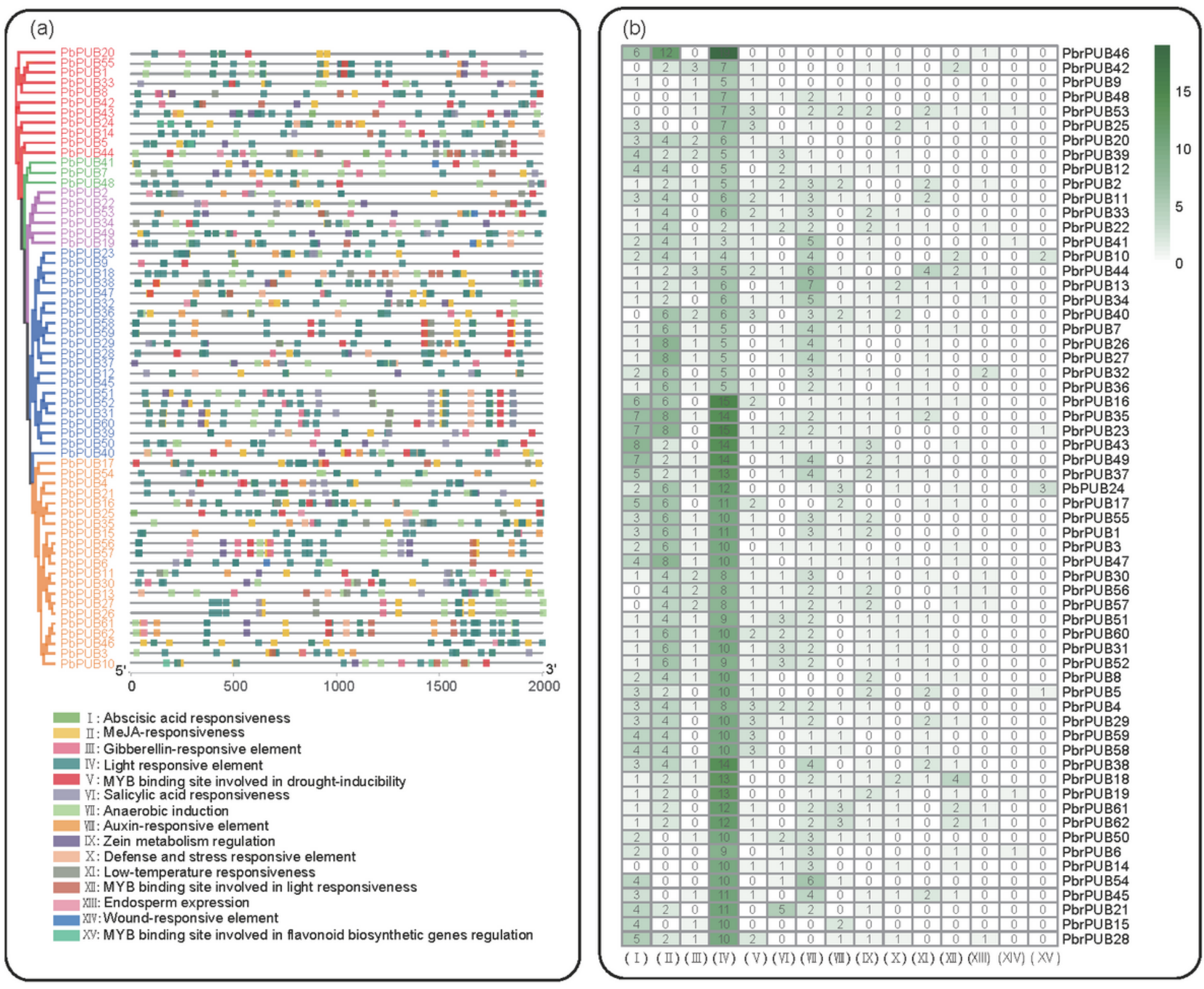

Figure 4

The cis-acting elements analysis of putative promoter of 62 PbrPUB genes. (a) The distribution pattern of 15 cis-acting elements of putative promoter of PUB gene family in pear. The phylogenetic tree was constructed by Mega-X using NJ method with 1000 bootstrap; (b) The number of 15 cis-acting elements of putative promoter of PbrPUB genes. The color scale at the top right indicated the number of cis-acting elements. Green color indicated the number of cis-acting elements on PUB member. 15 cis-acting elements including: (I) Abscisic acid responsiveness; (II) MeJA-responsiveness; (III) Gibberellin-responsive element; (IV) Light responsive element; (V) MYB binding site involved in drought-inducibility; (VI) Salicylic acid responsiveness; (VII) Anaerobic induction; (VIII) Auxin-responsive element; (IX) Zein metabolism regulation; $(X)$ Defense and stress responsive element; (XI) Low-temperature responsiveness; (XII) MYB binding site involved in light responsiveness; (XIII) Endosperm expression; (XIV) Wound-responsive element; (XV) MYB binding site involved in flavonoid biosynthetic genes regulation. 


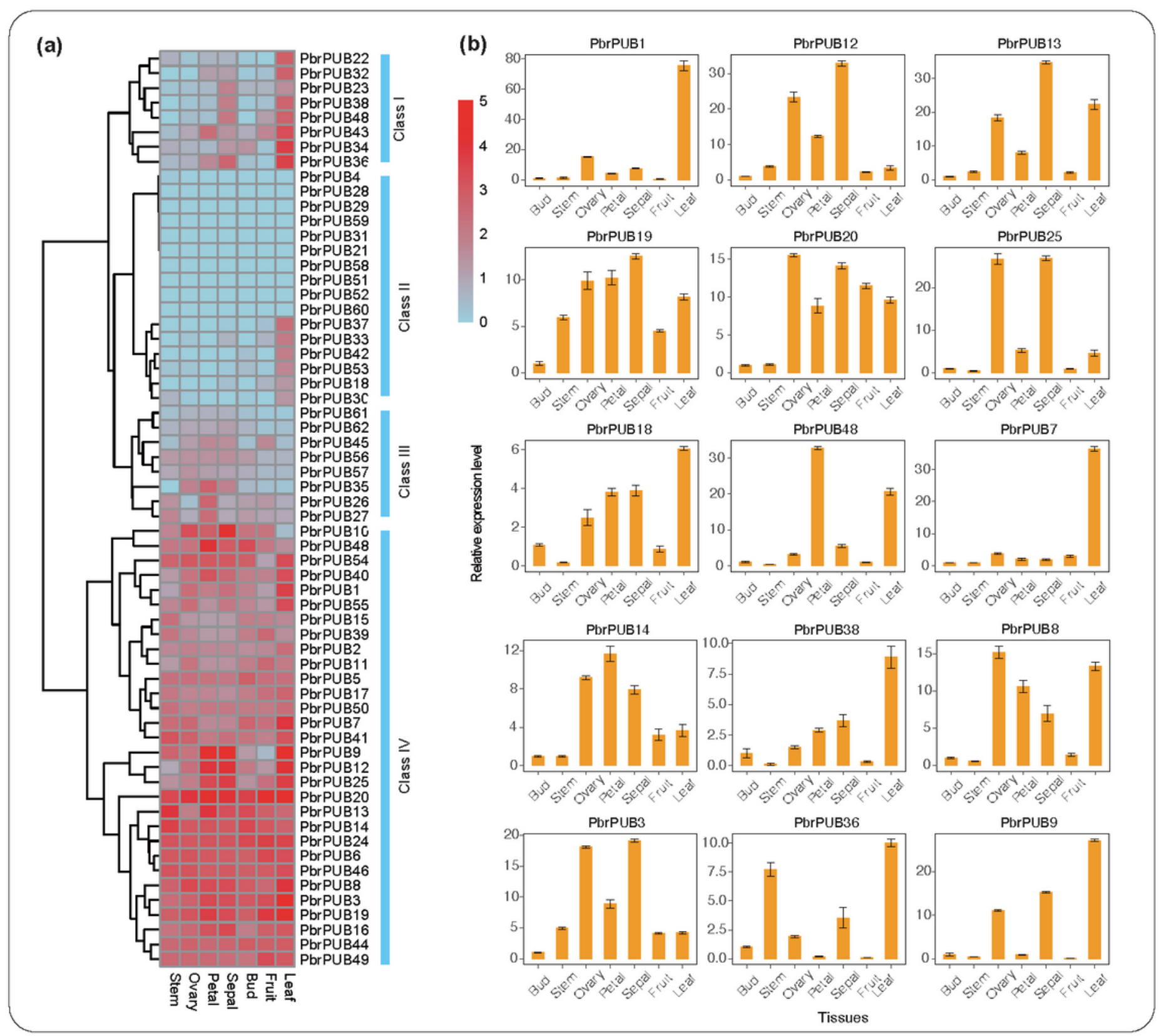

\section{Figure 5}

The expression pattern analysis of PUB gene family in seven different tissues. (a) The heatmap of expression level of PUB gene family in seven different tissues, including stem, ovary, petal, sepal, bud, fruit and leaf. Pheatmap, an R package, were used to generate the heatmap. The color scale represented the RPKM (reads per kilobase per million) values normalized by log2. Red color represented high expression, while blue represented low expression; (b) The expression levels of 15 randomly selected genes in seven different tissues were detected by qRT-PCR experiment. Seven tissues are comprised of bud, stem, ovary, petal, sepal, fruit and leaf. The x-axes represented seven different tissues; the $y$-axes represented the relatively expression of PUB genes. 

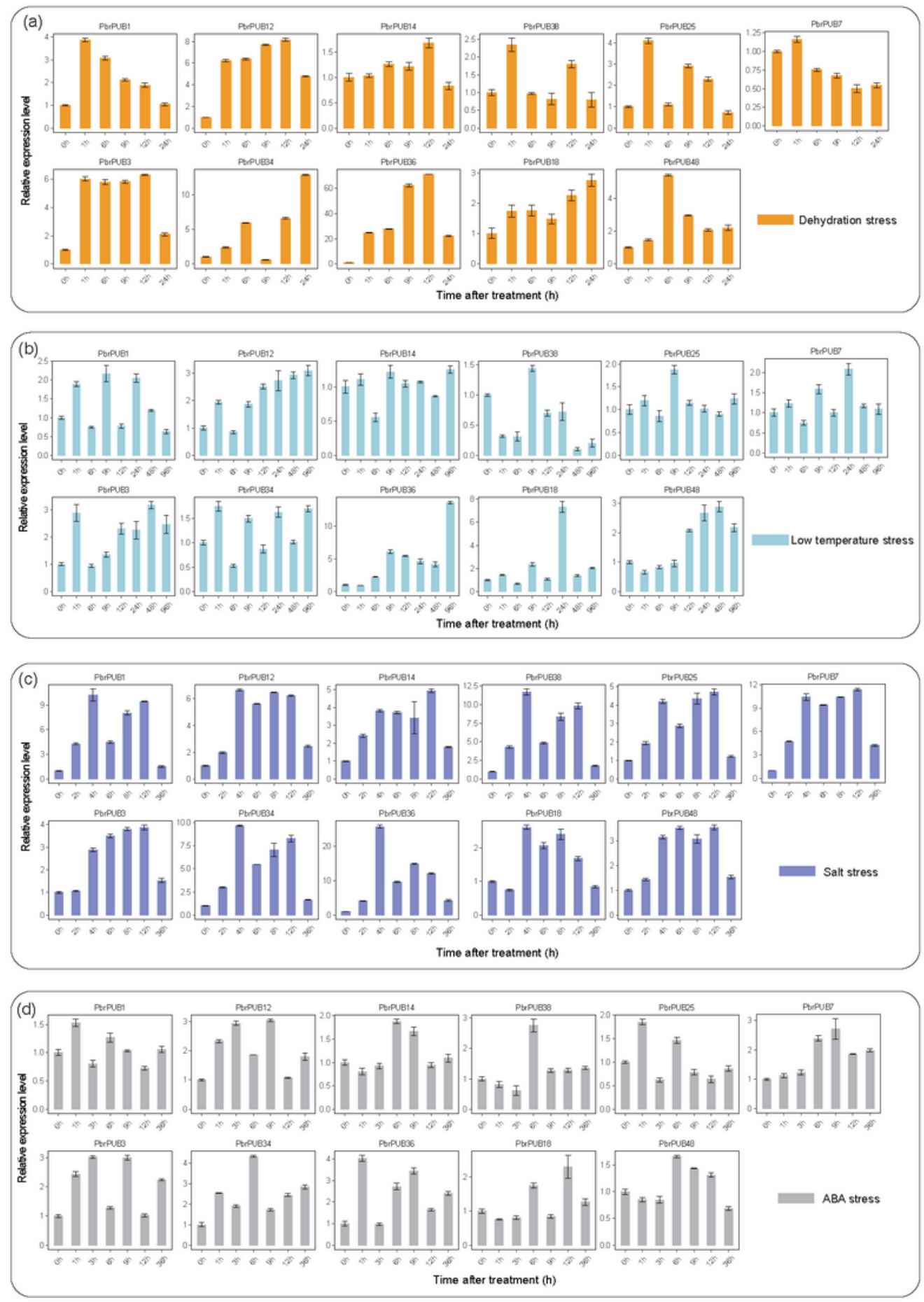

Figure 6

The expression level of 11 randomly selected PUB genes in four stresses were detected by qRT-PCR experiment. (a) For dehydration treatment, the shoots were placed on dry filter papers for $0,1,6,9,12$ and $24 \mathrm{~h}$; (b) For cold stress, the seedlings were placed in the chamber set at $4^{\circ} \mathrm{C}$ for $0,1,6,9,12,24,48$ and $96 \mathrm{~h}$; (c) For salt stress, the seedlings were placed in solution containing $200 \mathrm{mM} \mathrm{NaCl}$ solution for $0,2,4$, 6, 8, 12 and 36 h; (d) For ABA stress, The seedlings were dipped in solution containing $100 \mu \mathrm{M}$ ABA for 0, 
$1,3,6,9,12$ and $36 \mathrm{~h}$. The $\mathrm{x}$-axes represented time after treatment; the $\mathrm{y}$-axes represented the relatively expression of PUB genes.

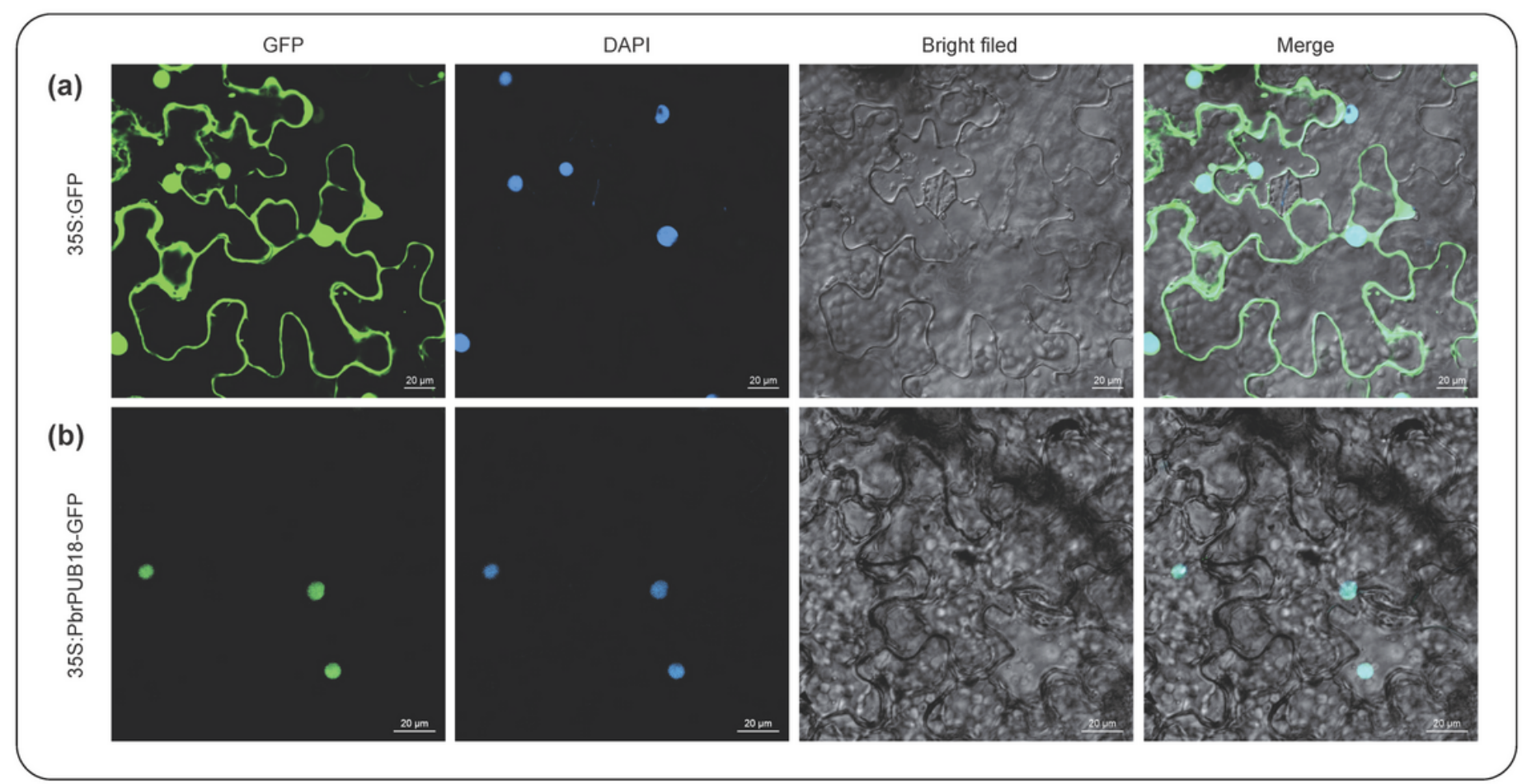

\section{Figure 7}

The Subcellular localization of PbrPUB18. (a) Tobacco leaf epidermal cells were transiently transformed with constructs containing 35S:GFP vector alone as control; (b) Transient expression of fusion plasmid (35S:PbrPUB18-GFP) in tobacco leaf epidermal cells. The nucleus was identified by DAPI staining. Green fluorescence images, DAPI staining mages, blight field images and the merged images are shown from left to right. Scale bars $=20 \mu \mathrm{m}$. 

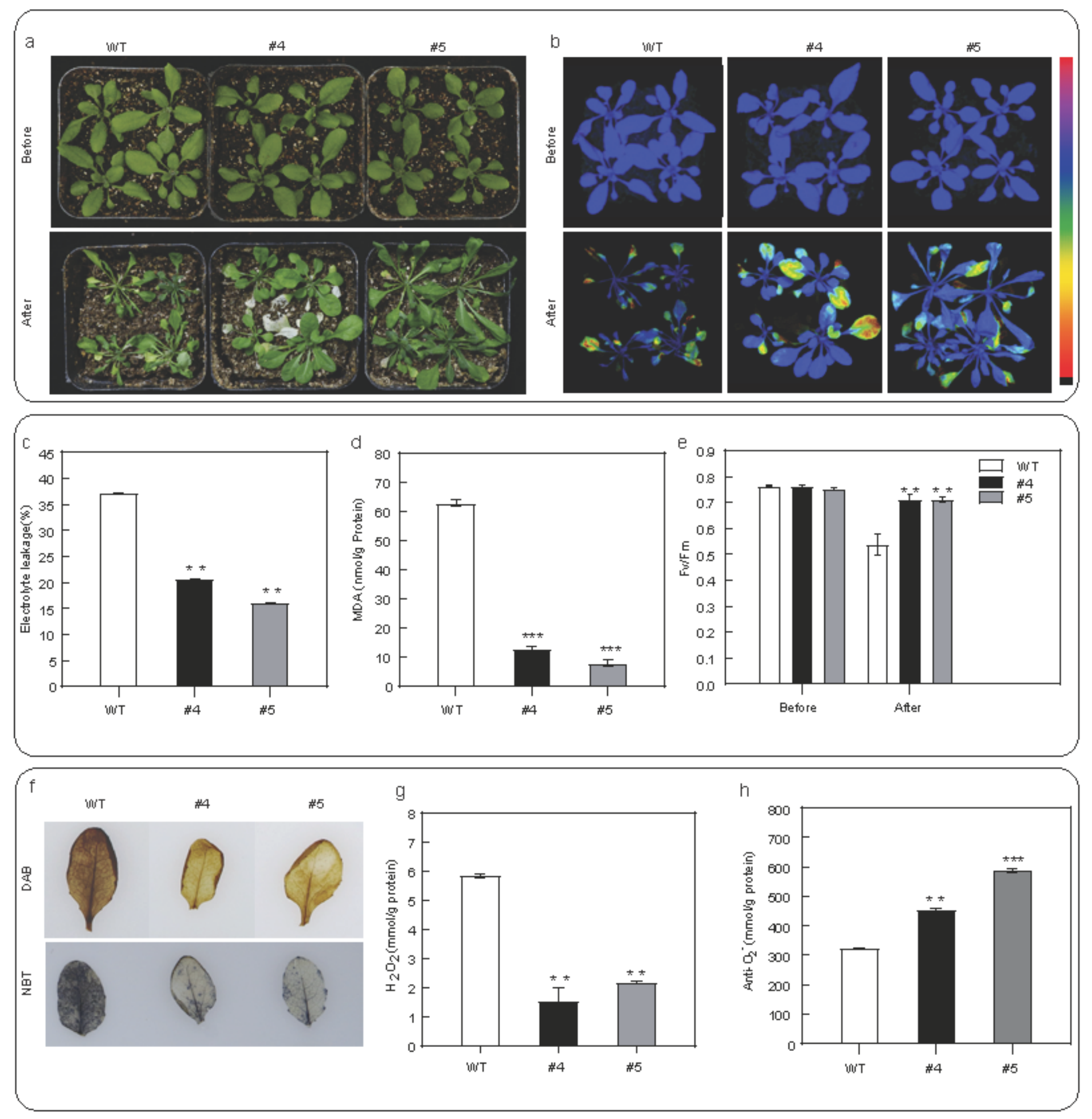

\section{Figure 8}

Drought tolerance assay of transgenic Arabidopsis plants overexpressing PbrPUB18. (a) Phenotypes of 15-day-old transgenic plants and WT before and after 12 days drought stress; (b) Images of (Fv/Fm). The false color code depicted on the right of the image ranges from 0 (black) to 1.0 (purple); Electrolyte leakage (c), MDA contents (d) in the WT, OE-4 and OE-5 after drought treatment; (e) Fv/Fm of WT, OE-4 and $\mathrm{OE}-5$ before and after drought stress; (f) Histochemical staining with DAB and NBT for detection of 
H2O2 and 02-, respectively, in WT, OE-4 and OE-5 after drought stress for 12 days; Levels of H2O2 (g) and anti-02- (h) in Arabidopsis WT, OE-4 and OE-5 after drought treatment. Asterisks indicate that the value is significantly different from that of the WT at the same time point $\left({ }^{* * P}<0.01 ; * \star * P<0.001\right)$.

\section{Supplementary Files}

This is a list of supplementary files associated with this preprint. Click to download.

- Table1.xlsx

- Additionalfile1Fig.S1.pdf

- Additionalfile2Fig.S2.pdf

- Additionalfile3FigS3.pdf

- Additionalfile4TableS1.xlsx 